\title{
Surfaces of revolution with vanishing curvature in Galilean 3-space
}

\author{
M. Dede ${ }^{\dagger}$, C. Ekici ${ }^{\ddagger}$, and W. Goemans` \\ ${ }^{\dagger}$ Department of Mathematics, Kilis 7 Aralık University, 79000 Kilis, \\ Turkey, mustafadede03@gmail.com \\ $\ddagger$ Department of Mathematics-Computer, Eskişehir Osmangazi \\ University, 26480 Eskişehir, Turkey, cumali.ekici@gmail.com \\ ${ }^{\star}$ Faculty of Economics and Business, KU Leuven, 1000 Brussels, \\ Belgium, wendy.goemans@kuleuven.be
}

June 18, 2017

\begin{abstract}
In this article, we define and study three types of surfaces of revolution in Galilean 3-space. The construction of the well-known surface of revolution, being the trace of a planar curve that is rotated about an axis in the supporting plane of the curve, is carried over to Galilean 3 -space. Then, we classify the surfaces of revolution with vanishing Gaussian curvature or vanishing mean curvature in Galilean 3-space. Depending on the type of surface of revolution, either the vanishing of the Gaussian curvature turns out to be equivalent to the vanishing of the mean curvature or one concludes immediately that there do not exist surfaces of revolution of that type with vanishing mean curvature.

Keywords: surface of revolution, rotational surface, flat surface, minimal surface, Galilean 3-space
\end{abstract}

MSC2010: 53A10, 53A35, 53A40

\section{Introduction}

Together with the ruled surfaces, the surfaces of revolution were one of the first study subjects in differential geometry, apart from curves. Being the trace of a rotated planar curve, surfaces of revolution are also found widely in the 'real world'. The catenoid, obtained by rotating a catenary, is one of the elementary minimal surfaces. Although 
surfaces of revolution or rotational surfaces are studied thoroughly already for centuries, we believe we can make a modest contribution to this research area by defining and studying them in Galilean 3-space. The geometry of this non-Euclidean space was first studied intensively by Röschel [11]. In the last decade, this space is used by several researchers as ambient space to translate to and investigate in well-known Euclidean concepts, see e.g. $[2,3,7,9,10]$ for examples on special surfaces. Investigations on surfaces in Galilean 3-space can also be found in $[1]$.

In [7], the author mentions two types of surfaces of revolution in Galilean 3-space as examples. It is pointed out there that those surfaces are Weingarten surfaces, that is, there exists a non-trivial functional dependence between the Gaussian curvature and the mean curvature. Further no systematic study of the curvatures of these surfaces is made. Since the authors of this article want to study twisted surfaces, which are surfaces that are traced out by a planar curve on which two simultaneous rotations are performed and hence which are a generalization of surfaces of revolution (see [6] and the references therein), in a subsequent work [4], first the present investigation is carried out. To the best of our knowledge this has not been done before.

In this article we first recall the necessary preliminaries from Galilean space. Then we define three different types of surfaces of revolution in that space and examine curvature properties of those surfaces. We present classification theorems of surfaces of revolution with vanishing Gaussian curvature or vanishing mean curvature.

\section{Preliminaries}

For an in depth study of the Galilean 3-space, see [11]. Here, we recall the properties that we need from that work.

The Galilean 3 -space $\mathbb{G}^{3}$ originates in a Cayley-Klein way by pointing out an absolute figure $\{\omega, f, I\}$ in the 3-dimensional real projective space. Here $\omega$ is the absolute plane, $f$ the absolute line and $I$ the fixed elliptic involution of points of $f$. Then homogeneous coordinates $\left(x_{0}: x_{1}: x_{2}: x_{3}\right)$ are introduced such that $\omega$ is given by $x_{0}=0, f$ by $x_{0}=x_{1}=0$ and $I$ by $\left(0: 0: x_{2}: x_{3}\right) \mapsto\left(0: 0: x_{3}:-x_{2}\right)$. The group of motions of $\mathbb{G}^{3}$ is a six-parameter group. Regarding this group of motions, apart from the absolute plane, there exist two classes of planes in $\mathbb{G}^{3}$ : Euclidean planes which contain $f$ and in which the induced metric is Euclidean and isotropic planes that do not contain $f$ and in which the induced metric is isotropic. Also, there are four types of lines in $\mathbb{G}^{3}$ : isotropic lines which intersect $f$, non-isotropic lines which do not intersect $f$, non-isotropic lines in $\omega$ and the absolute line $f$.

In affine coordinates defined by $\left(x_{0}: x_{1}: x_{2}: x_{3}\right)=\left(1: x_{1}: x_{2}:\right.$ 
$\left.x_{3}\right)$, the distance between two points $P_{i}=\left(x_{i}, y_{i}, z_{i}\right)$ with $i \in\{1,2\}$ is defined by

$$
d\left(P_{1}, P_{2}\right)=\left\{\begin{array}{cl}
\left|x_{2}-x_{1}\right| & \text { if } x_{1} \neq x_{2}, \\
\sqrt{\left(y_{2}-y_{1}\right)^{2}+\left(z_{2}-z_{1}\right)^{2}} & \text { if } x_{1}=x_{2} .
\end{array}\right.
$$

A vector $\vec{a}=(x, y, z)$ is isotropic if $x=0$ and non-isotropic otherwise. Hence for standard coordinates $(x, y, z)$, the $x$-axis is nonisotropic while the $y$-axis and the $z$-axis are isotropic. The $y z$-plane, $x=0$, is Euclidean and the $x y$-plane and the $x z$-plane are isotropic. The Galilean scalar product of two vectors $\vec{a}=(x, y, z)$ and $\vec{b}=$ $\left(x_{1}, y_{1}, z_{1}\right)$ is defined by

$$
\langle\vec{a}, \vec{b}\rangle=\left\{\begin{array}{cl}
x x_{1} & \text { if } x \neq 0 \text { or } x_{1} \neq 0 \\
y y_{1}+z z_{1} & \text { if } x=x_{1}=0
\end{array}\right.
$$

A vector $\vec{a}$ is a unit vector if $\|\vec{a}\|:=\sqrt{\langle\vec{a}, \vec{a}\rangle}=1$. The Galilean cross product of two vectors $\vec{a}=(x, y, z)$ and $\vec{b}=\left(x_{1}, y_{1}, z_{1}\right)$ is defined as

$$
\vec{a} \wedge \vec{b}=\left|\begin{array}{ccc}
0 & e_{2} & e_{3} \\
x & y & z \\
x_{1} & y_{1} & z_{1}
\end{array}\right| .
$$

In order to define surfaces of revolution we need the two types of rotations in $\mathbb{G}^{3}$. A Euclidean rotation about the non-isotropic $x$-axis is given by

$$
\left(\begin{array}{l}
x^{\prime} \\
y^{\prime} \\
z^{\prime}
\end{array}\right)=\left(\begin{array}{ccc}
1 & 0 & 0 \\
0 & \cos \theta & \sin \theta \\
0 & -\sin \theta & \cos \theta
\end{array}\right)\left(\begin{array}{l}
x \\
y \\
z
\end{array}\right)
$$

where $\theta$ is the Euclidean angle. An isotropic rotation is given by

$$
\left(\begin{array}{l}
x^{\prime} \\
y^{\prime} \\
z^{\prime}
\end{array}\right)=\left(\begin{array}{lll}
1 & 0 & 0 \\
\theta & 1 & 0 \\
0 & 0 & 1
\end{array}\right)\left(\begin{array}{l}
x \\
y \\
z
\end{array}\right)+\left(\begin{array}{c}
c \theta \\
c \\
\frac{c}{2} \theta^{2} \\
0
\end{array}\right)
$$

where $\theta$ is the isotropic angle and $c \in \mathbb{R}_{0}$. Here the bundle of fixed planes is given by $z=$ constant.

Finally, in order to be able to calculate curvatures, we have to be able to perform patch computations for a surface in $\mathbb{G}^{3}$. If a surface in $\mathbb{G}^{3}$ is parameterized by

$$
\varphi\left(v^{1}, v^{2}\right)=\left(x\left(v^{1}, v^{2}\right), y\left(v^{1}, v^{2}\right), z\left(v^{1}, v^{2}\right)\right)
$$

then denote the first order derivatives for $i \in\{1,2\}$ by $\varphi_{, i}=\frac{\partial \varphi}{\partial v^{i}}\left(v^{1}, v^{2}\right)$. Here we always assume that the surfaces are admissible, that is, its tangent plane is nowhere a Euclidean plane. 
The unit normal vector $N$ of the surface is defined by

$$
N=\frac{\varphi, 1 \wedge \varphi_{, 2}}{w} \quad \text { where } w=\left\|\varphi_{, 1} \wedge \varphi_{, 2}\right\| .
$$

The coefficients of the second fundamental form are given by

$$
L_{i j}=\left\langle\frac{\varphi_{, i j} x_{, 1}-x_{, i j} \varphi_{, 1}}{x_{, 1}}, N\right\rangle=\left\langle\frac{\varphi_{, i j} x_{, 2}-x_{, i j} \varphi_{, 2}}{x_{, 2}}, N\right\rangle .
$$

The Gaussian curvature $K$ and the mean curvature $H$ of the surface are defined in [11], analogous to Euclidean space, by

$$
K=\frac{L_{11} L_{22}-L_{12}^{2}}{w^{2}} \quad \text { and } \quad 2 H=\sum_{i, j=1}^{2} g^{i j} L_{i j}
$$

where

$$
g^{1}=\frac{x_{, 2}}{w}, g^{2}=-\frac{x_{, 1}}{w} \text { and } g^{i j}=g^{i} g^{j} \text { for } i, j \in\{1,2\} .
$$

As proved in [11] (Satz 19.5, page 107), the mean curvature in a point $p$ of a surface in $\mathbb{G}^{3}$ is the curvature of the intersection of the surface with the Euclidean plane that contains the point $p$.

\section{Surfaces of revolution in Galilean 3-space}

We construct a surface of revolution in Galilean 3-space analogously to how that is done in Euclidean 3-space.

Definition 3.1. A surface of revolution in $\mathbb{G}^{3}$ is a surface that is traced out by a planar curve, the profile curve, that is subjected to a rotation in $\mathbb{G}^{3}$. This rotation is either a Euclidean rotation about an axis in the supporting plane of the profile curve, or an isotropic rotation for which a choice of a bundle of fixed planes is made.

Because of the existence of the different kinds of planes in $\mathbb{G}^{3}$, we consider two possibilities for the supporting plane of the profile curve of a surface of revolution in $\mathbb{G}^{3}$ : either the profile curve lies in a Euclidean plane or it lies in an isotropic plane.

Since a Euclidean plane only contains isotropic vectors while an isotropic plane contains both isotropic and non-isotropic vectors, there are three types of surfaces of revolution to be defined in $\mathbb{G}^{3}$.

\subsection{Type I surfaces of revolution in $\mathbb{G}^{3}$}

Without losing generality, we can assume that the profile curve $\alpha$ lies in the Euclidean $y z$-plane and is parameterized by $\alpha(t)=(0, f(t), g(t))$ 
with $f$ and $g$ real functions. On this profile curve we perform an isotropic rotation with $c \in \mathbb{R}_{0}$, for instance,

$$
\left(\begin{array}{lll}
1 & 0 & 0 \\
s & 1 & 0 \\
0 & 0 & 1
\end{array}\right)\left(\begin{array}{c}
0 \\
f(t) \\
g(t)
\end{array}\right)+\left(\begin{array}{c}
c s \\
c \\
\frac{2}{2} s^{2} \\
0
\end{array}\right) .
$$

Thus, up to a transformation, a type I surface of revolution in $\mathbb{G}^{3}$ is parameterized by

$$
\varphi(s, t)=\left(c s, f(t)+\frac{c}{2} s^{2}, g(t)\right) .
$$

These type I surfaces of revolution are one of the two kinds of surfaces of revolution that are mentioned as examples in [7].

\subsection{Type II surfaces of revolution in $\mathbb{G}^{3}$}

Now we assume, again without losing generality, that the profile curve $\alpha$ lies in the isotropic $x y$-plane and is parameterized by $\alpha(t)=(f(t)$, $g(t), 0)$ with $f$ and $g$ real functions. Also on this profile curve we perform an isotropic rotation with $c \in \mathbb{R}_{0}$, this time,

$$
\left(\begin{array}{lll}
1 & 0 & 0 \\
0 & 1 & 0 \\
s & 0 & 1
\end{array}\right)\left(\begin{array}{c}
f(t) \\
g(t) \\
0
\end{array}\right)+\left(\begin{array}{c}
c s \\
0 \\
c s^{2}
\end{array}\right) .
$$

Then, up to a transformation, a type II surface of revolution in $\mathbb{G}^{3}$ is parameterized by

$$
\varphi(s, t)=\left(f(t)+c s, g(t), s f(t)+\frac{c}{2} s^{2}\right) .
$$

As far as we know, this type of surfaces of revolution in $\mathbb{G}^{3}$ has not been defined before.

\subsection{Type III surfaces of revolution in $\mathbb{G}^{3}$}

Finally, again we start with a profile curve $\alpha(t)=(f(t), g(t), 0)$ in the isotropic $x y$-plane, with $f$ and $g$ real functions, but this time we perform a Euclidean rotation about the $x$-axis on it,

$$
\left(\begin{array}{ccc}
1 & 0 & 0 \\
0 & \cos s & \sin s \\
0 & -\sin s & \cos s
\end{array}\right)\left(\begin{array}{c}
f(t) \\
g(t) \\
0
\end{array}\right) \text {. }
$$

So, up to a transformation, a type III surface of revolution in $\mathbb{G}^{3}$ is parameterized by

$$
\varphi(s, t)=(f(t), g(t) \cos s,-g(t) \sin s) .
$$

These type III surfaces of revolution are also used as examples in [7]. 


\section{Zero curvature surfaces of revolution in $\mathbb{G}^{3}$}

For the three types of surfaces of revolution in $\mathbb{G}^{3}$ we calculate the Gaussian curvature and the mean curvature and we examine when these vanish. As in Euclidean 3-space, we call surfaces with identically vanishing Gaussian curvature flat and surfaces with identically vanishing mean curvature minimal. From [11] we mention the following important theorem which classifies all minimal surfaces in $\mathbb{G}^{3}$.

Theorem 4.1 ([11]). The minimal surfaces in Galilean 3-space are cones whose vertex lies on the absolute line $f$ and type $C$ ruled surfaces, that is, conoidal surfaces having the absolute line $f$ as the directional line at infinity.

A type $\mathrm{C}$ ruled surface can be parameterized as follows

$$
\varphi(s, t)=(s, f(s), 0)+t\left(0, \beta_{2}(s), \beta_{3}(s)\right)
$$

with $f, \beta_{2}$ and $\beta_{3}$ at least three times continuous differentiable real functions such that $\beta_{2}(s)^{2}+\beta_{3}(s)^{2}=1$.

\subsection{Zero curvature type I surfaces of revolution in $\mathbb{G}^{3}$}

For a type I surface of revolution in $\mathbb{G}^{3}$, parametrized by

$$
\varphi(s, t)=\left(c s, f(t)+\frac{c}{2} s^{2}, g(t)\right)
$$

with $c \in \mathbb{R}_{0}$, using (1), one easily calculates

$$
K=\frac{g^{\prime}\left(f^{\prime \prime} g^{\prime}-f^{\prime} g^{\prime \prime}\right)}{c\left(f^{\prime 2}+g^{\prime 2}\right)^{2}} \quad \text { and } \quad H=\operatorname{sgn}(c) \frac{f^{\prime} g^{\prime \prime}-f^{\prime \prime} g^{\prime}}{2\left(f^{\prime 2}+g^{\prime 2}\right)^{3 / 2}} .
$$

Here and in the remainder of the paper we often drop the parameter of the functions $f$ and $g$ for reasons of readability. Primes denote derivation and by sgn we mean the sign function. Remark that $f^{\prime}$ and $g^{\prime}$ can not be both identically zero since then the surface is not admissible.

Further, it is immediate that the Gaussian curvature is identically zero if and only if the mean curvature is identically zero. Hence, a type I surface of revolution in $\mathbb{G}^{3}$ is flat if and only if it is minimal. Then the following classification theorem is valid.

Theorem 4.2. A type I surface of revolution in Galilean 3-space is flat or, equivalently, minimal, if and only if it is either 
1. a parabolic cylinder parameterized by

$$
\varphi(s, t)=\left(c s, a+\frac{c}{2} s^{2}, g(t)\right),
$$

2. a part of an isotropic plane, consisting of a family of parabolas, parameterized by

$$
\varphi(s, t)=\left(c s, f(t)+\frac{c}{2} s^{2}, a\right),
$$

3. a parabolic cylinder parameterized by

$$
\varphi(s, t)=\left(c s, f(t)+\frac{c}{2} s^{2}, a f(t)+b\right) .
$$

Here $a, b, c \in \mathbb{R}$ with $c \neq 0$ and $a \neq 0$ in parameterization (4).

Proof. From the expressions for the Gaussian curvature and the mean curvature it is clear that it is sufficient that $f^{\prime} g^{\prime \prime}-f^{\prime \prime} g^{\prime}=0$.

If $f^{\prime}=0$ or $g^{\prime}=0$, then we obtain parameterization (2) or (3) respectively.

If $f^{\prime} \neq 0$ and $g^{\prime} \neq 0$ one has $\frac{f^{\prime \prime}}{f^{\prime}}=\frac{g^{\prime \prime}}{g^{\prime}}$ and integrating this equation gives $g(t)=a f(t)+b$, with real integration constants $a \neq 0$ and $b$. Therefore, the profile curve is an isotropic straight line and this leads to parameterization (4).

Conversely, it is calculated immediately that the surfaces given by the parameterizations in the statement are flat and minimal.

The following corollary is immediate.

Corollary 4.3. A type I surface of revolution in Galilean 3-space is flat, or equivalently minimal, if and only if its profile curve is an isotropic straight line.

Remark. It is easy to see that in parameterization (2) the components satisfy $y=a+\frac{x^{2}}{2 c}$. Similarly, in parameterization (4) a straightforward calculation shows that $y=\frac{z-b}{a}+\frac{x^{2}}{2 c}$.

Moreover, reparameterize $(2)$ such that $g(t)=v$. Then the resulting parameterization describes a type $\mathrm{C}$ ruled surface,

$$
\varphi(s, v)=\left(c s, a+\frac{c}{2} s^{2}, 0\right)+v(0,0,1) .
$$

One can proceed similarly for parameterization (3). Also, reparameterize (4) such that $f(t)=v$, then it becomes

$$
\varphi(s, v)=\left(c s, \frac{c}{2} s^{2}, b\right)+v(0,1, a) .
$$


Thus, this leads to a conoidal surface having the absolute line $f$ as the directional line at infinity, hence, a type $\mathrm{C}$ ruled surface. For a drawing of a surface parameterized by parameterization (4), see Figure 1(a).

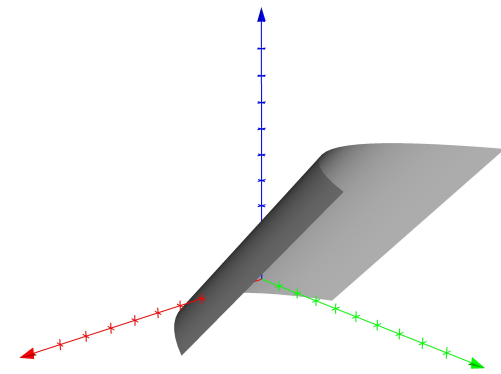

(a) A flat and minimal type I surface of revolution, parameterized by (4) with $a=1, b=c=2$ and $f$ any function.

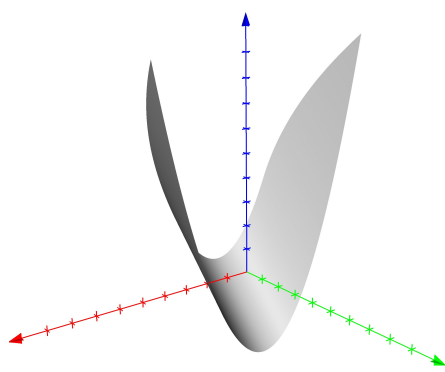

(b) A flat and minimal type II surface of revolution, parameterized by (6) with $a=c=1$ and $b=-2$.

Figure 1: Flat and minimal type I and type II surfaces of revolution.

Remark. As mentioned in [7], since the Gaussian curvature and the mean curvature of type I surfaces of revolution are functions of one variable only, these type I surfaces of revolution are Weingarten surfaces. Indeed, $\operatorname{sgn}(c) c\left(f^{\prime 2}+g^{\prime 2}\right)^{1 / 2} K=2 g^{\prime} H$.

\subsection{Zero curvature type II surfaces of revolution in} $\mathbb{G}^{3}$

A type II surface of revolution in $\mathbb{G}^{3}$ parametrized by

$$
\varphi(s, t)=\left(f(t)+c s, g(t), s f(t)+\frac{c}{2} s^{2}\right)
$$

with $c \in \mathbb{R}_{0}$, using (1), is calculated to have Gaussian curvature and mean curvature

$$
K=\frac{c^{2} g^{\prime}}{w^{4}}\left[f\left(f^{\prime} g^{\prime \prime}-f^{\prime \prime} g^{\prime}\right)-f^{\prime 2} g^{\prime}\right]
$$

and

$$
H=\frac{c^{2}}{2 w^{3}}\left[f\left(f^{\prime} g^{\prime \prime}-f^{\prime \prime} g^{\prime}\right)-f^{\prime 2} g^{\prime}\right] .
$$

Here $w^{2}=f^{2} f^{\prime 2}+c^{2} g^{\prime 2}$. Again, remark that $f^{\prime}$ and $g^{\prime}$ can not be both identically zero since then the surface is not admissible.

Also now, the Gaussian curvature is identically zero if and only if the mean curvature is identically zero. Thus, a type II surface of 
revolution in $\mathbb{G}^{3}$ is flat if and only if it is minimal. Similar as for the type I surfaces of revolution the following classification theorem can be proved.

Theorem 4.4. A type II surface of revolution in Galilean 3-space is flat or, equivalently, minimal, if and only if it is either

1. a part of an isotropic plane, consisting of family of parabolas, parameterized by

$$
\varphi(s, t)=\left(f(t)+c s, a, s f(t)+\frac{c}{2} s^{2}\right),
$$

2. a parabolic cylinder parameterized by

$$
\varphi(s, t)=\left(a+c s, g(t), a s+\frac{c}{2} s^{2}\right),
$$

3. a cyclic surface (parabolic sphere) parameterized by

$$
\varphi(s, t)=\left(t+c s, a t^{2}+b, s t+\frac{c}{2} s^{2}\right),
$$

where $a, b, c \in \mathbb{R}$ and $c \neq 0$.

In order to find parameterization (6) in Theorem 4.4 we assume that the profile curve is parameterized by arclength, then $f(t)=t$.

Remark. A straightforward calculation shows that the components of parameterization (5) satisfy

$$
z=\frac{x^{2}}{2 c}-\frac{a^{2}}{2 c} .
$$

Similarly, for the components of parameterization (6) one sees that

$$
x^{2}-\frac{y}{a}-2 c z+\frac{b}{a}=0 .
$$

Again, reparameterize (5) using $g(t)=v$, then it is seen to be a conoidal surface that has the absolute line $f$ as the directional line at infinity, hence, it is a type $\mathrm{C}$ ruled surface,

$$
\varphi(s, v)=\left(a+c s, 0, a s+\frac{c}{2} s^{2}\right)+v(0,1,0) .
$$

Similar for parameterization (6), since setting $u=t+c s$ and $v=$ $-s\left(t+\frac{c}{2} s\right)$ reduces it to

$$
\varphi(u, v)=\left(u, a u^{2}+b, 0\right)+v(0, a c,-1) .
$$


Remark that in parameterization (6) the profile curve is an isotropic circle, see [11]. In Galilean space this parameterization represents a cyclic surface constructed analogously to a Euclidean sphere, see [12]. For a drawing of such a surface, see Figure 1(b).

Remark. Also for type II surfaces of revolution the Gaussian curvature and the mean curvature are functions of one variable only, therefore, type II surfaces of revolution are Weingarten surfaces. Indeed, $w K=$ $2 g^{\prime} H$.

\subsection{Zero curvature type III surfaces of revolution in $\mathbb{G}^{3}$}

For a type III surface of revolution in $\mathbb{G}^{3}$ given by the parametrization

$$
\varphi(s, t)=(f(t), g(t) \cos s,-g(t) \sin s),
$$

using (1), it is calculated that

$$
K=\frac{f^{\prime \prime} g^{\prime}-f^{\prime} g^{\prime \prime}}{f^{\prime 3} g} \quad \text { and } \quad H=\frac{\operatorname{sgn}\left(f^{\prime} g\right)}{2 g} .
$$

Here $f^{\prime}$ should be non-zero in order to have an admissible surface. Also the function $g$ must be non-zero of course.

For these type III surfaces of revolution in $\mathbb{G}^{3}$ the flat and minimal conditions are not equivalent. Moreover, it is immediate that there do not exist minimal type III surfaces of revolution in $\mathbb{G}^{3}$.

This time a similar proof as that of Theorem 4.2 leads to the classification of flat type III surfaces of revolution in $\mathbb{G}^{3}$.

Theorem 4.5. A type III surface of revolution in Galilean 3-space is flat if and only if it is either

1. a cylinder over a Euclidean circle, parameterized by

$$
\varphi(s, t)=(f(t), a \cos s,-a \sin s)
$$

2. a circular cone with vertex $(b, 0,0)$, parameterized by

$$
\varphi(s, t)=(a g(t)+b, g(t) \cos s,-g(t) \sin s),
$$

where $a, b \in \mathbb{R}$ with $a \neq 0$.

Remark. We can reparameterize (7) by implementing $f(t)=v$, to

$$
\varphi(s, v)=(0, a \cos s,-a \sin s)+v(1,0,0) .
$$

In [11], a surface of this kind is called a type B ruled surface. 
Remark. As mentioned in [7], it is immediate that, since the Gaussian curvature and the mean curvature of type III surfaces of revolution are functions of one variable only, type III surfaces of revolution are Weingarten surfaces. Indeed, $\operatorname{sgn}\left(f^{\prime} g\right) f^{\prime 2} K=2\left(f^{\prime \prime} g^{\prime}-f^{\prime} g^{\prime \prime}\right) H$.

Only for type III surfaces of revolution it is possible to determine immediately which ones have constant mean curvature. In that case the function $g$ is a non-zero constant function, hence, it is also a flat surface, more specific a cylinder over a Euclidean circle as in parameterization (7) of Theorem 4.5.

\section{Conclusion and further research}

In this work we defined three types of surfaces of revolution in Galilean 3 -space and examined when these are flat or minimal. For type I and type II surfaces the flat and minimal conditions are equivalent. There do not exist minimal type III surfaces of revolution in $\mathbb{G}^{3}$.

Similar to how Minkowski 3-space relates to Euclidean 3-space, one has the notion of pseudo-Galilean 3 -space $\mathbb{G}_{1}^{3}$. Without going into detail here, $\mathbb{G}_{1}^{3}$ is similar to $\mathbb{G}^{3}$ but the pseudo-Galilean scalar product of two vectors $\vec{a}=(x, y, z)$ and $\vec{b}=\left(x_{1}, y_{1}, z_{1}\right)$ is defined by

$$
\langle\vec{a}, \vec{b}\rangle=\left\{\begin{array}{cl}
x x_{1} & \text { if } x \neq 0 \text { or } x_{1} \neq 0 \\
y y_{1}-z z_{1} & \text { if } x=x_{1}=0
\end{array}\right.
$$

Because of this there exist four types of isotropic vectors $\vec{a}=(0, y, z)$ in $\mathbb{G}_{1}^{3}$ : spacelike ones (if $y^{2}-z^{2}>0$ ), timelike ones (if $y^{2}-z^{2}<0$ ) and two types of lightlike ones (if $y= \pm z$ ). Analougously as in Minkowski 3 -space, instead of the trigonometric functions the hyperbolic functions must be used to describe rotations. Therefore it is clear that one can also define different types of surfaces of revolution in $\mathbb{G}_{1}^{3}$. This is done in [8] but it seems that one type is missing there. Hence the completion of this could be the subject of further research, as well as a more elaborate study of the constancy of the curvatures of surfaces of revolutions and this in both Galilean and pseudo-Galilean 3-space. See also [5] and [13], in the latter the Gauss map of two types of surfaces of revolution in $\mathbb{G}_{1}^{3}$ is studied.

The drawings in this article are made with VisuMath, for more information see www.visumath.be.

\section{Acknowledgements}

The authors wish to thank the referee for the valuable comments that improved the first version of the paper. Especially also for pointing out the existence of reference [1]. 


\section{References}

[1] A. Artykbaev and D.D. Sokolov Geometry in the large in a flat space-time, Tashkent, Publ. House FAN Uzbekian SSR, 1991. (Russian)

[2] M. Dede, Tubular surfaces in Galilean space, Math. Commun. 18 (2013), 209-217.

[3] M. Dede, C. Ekici and A. Ceylan Çöken, On the parallel surfaces in Galilean space, Hacet. J. Math. Stat. 42(6) (2013), 605-615.

[4] M. Dede, C. Ekici, W. Goemans and Y. Ünlütürk, Twisted surfaces with vanishing curvature in Galilean 3-space, preprint.

[5] B. Divjak and Ž. Milin Šipuš, Some special surfaces in the pseudo-Galilean space, Acta Math. Hung. 118(3) (2008), 209 226.

[6] W. Goemans and I. Van de Woestyne, Twisted surfaces with null rotation axis in Minkowski 3-space, Results Math. 70(1) (2016), 81-93 (doi:10.1007/s00025-015-0462-2).

[7] Ž. Milin Šipuš, Ruled Weingarten surfaces in Galilean space, Period. Math. Hung. 56(2) (2008), 213-225.

[8] Ž. Milin Šipuš and B. Divjak, Surfaces of constant curvature in the pseudo-Galilean space, Internat. J. Math. Math. Sci. (2012) doi:10.1155/2012/375264 (Article ID 375264).

[9] Ž. Milin Šipuš and B. Divjak, Translation surfaces in the Galilean space, Glas. Mat. Ser. III 46(66) (2011), 455-469.

[10] D. Palman, Drehzykliden des Galileischen Raumes $G_{3}$, Math. Pannon. 2(1) (1990), 95-104.

[11] O. Röschel, Die Geometrie des Galileischen Raumes, Bericht der Mathematisch-Statistischen Sektion in der Forschungsgesellschaft Joanneum, Bericht Nr. 256, Habilitationsschrift, Leoben, 1984.

[12] I. M. Yaglom, A simple non-Euclidean geometry and its physical basis, Springer-Verlag New York Inc, 1979.

[13] D. W. Yoon, Surfaces of revolution in the three dimensional pseudo-Galilean space, Glas. Mat. Ser. III 48(68) (2013), 415428. 